This is a postprint of the article Bayesian population correlation: A probabilistic approach to inferring and comparing population distributions for detrital zircon ages by Alex Tye (alex.tye@dixie.edu, @alexrtye), Aaron Wolf (aswolf@umich.edu), and Nathan Niemi (naniemi@umich.edu) which was published in Chemical Geology (https://doi.org/10.1016/j.chemgeo.2019.03.039).

This is the accepted version of the article, which may differ slightly from the final publication version, and is submitted to EarthArXiv. 


\title{
Bayesian Population Correlation: A probabilistic approach to inferring and comparing population distributions for detrital zircon ages
}

\author{
A. R. Tye ${ }^{a}$, A. S. Wolf ${ }^{a}$, N. A. Niemi ${ }^{\mathrm{a}}$ \\ ${ }^{a}$ University of Michigan, Ann Arbor, Department of Earth and Environmental Sciences, \\ $M I 48109$
}

\begin{abstract}
Populations of detrital zircons are shaped by geologic factors such as sediment transport, erosion mechanisms, and the zircon fertility of source areas. Zircon $\mathrm{U}-\mathrm{Pb}$ age datasets are influenced both by these geologic factors and by the statistical effects of sampling. Such statistical effects introduce significant uncertainty into the inference of parent population age distributions from detrital zircon samples. This uncertainty must be accounted for in order to understand which features of sample age distributions are attributable to earth processes and which are sampling effects. Sampling effects are likely to be significant at a range of common detrital zircon sample sizes (particularly when $n \lesssim 300$ ).

In order to more accurately account for the uncertainty in estimating parent population age distributions, we introduce a new method to infer probability model ensembles (PMEs) from detrital zircon samples. Each PME represents a set of the potential parent populations that are likely to have produced a given zircon age sample. PMEs form the basis of a new metric of correspondence between two detrital zircon samples, Bayesian Population Correlation (BPC), which is shown in a suite of numerical experiments to be unbiased with respect to sample size. BPC uncertainties can be directly estimated for a specific sample comparison, and BPC results conform to analytical predictions when comparing populations with known proportions of shared ages. We implement all of these features in a set of MATLAB $\mathbb{R}$ scripts made freely available as open-source code and as a standalone application. The robust uncertainties, lack of sample size bias, and predictability of $\mathrm{BPC}$ are desirable features that differentiate it from existing detrital zircon correspondence metrics. Additionally, analysis of other sample limited
\end{abstract}


datasets with complex probability distributions may also benefit from our approach.

Keywords:

probability, Bayesian, provenance, geochronology, density estimation

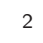

3

\section{Introduction}

Detrital zircon $\mathrm{U}-\mathrm{Pb}$ ages can provide a robust indicator of the provenance of sedimentary rocks or modern sediment through comparison with the ages of potential source rocks. The population of zircon ages in a sedimentary rock or modern sedimentary environment depends on source ages, zircon fertility of source areas, spatial and temporal variations in erosion, and sediment transport processes in the catchment (e.g., Amidon et al., 2005; Dickinson, 2008; Tranel et al., 2011; Gehrels, 2012; Satkoski et al., 2013; Garçon and Chauvel, 2014). Often, these zircon age populations are multi-modal, with the number and distribution of peaks unknown. A set of detrital zircon U$\mathrm{Pb}$ age measurements thus reflects the influence of earth processes, operator choices about which part of a grain to analyze (e.g., Hanchar and Miller, 1993), and the statistical effects of random sampling. Given the complexity of many detrital zircon age distributions, sampling effects can be significant and it is important to consider the method by which parent population characteristics are inferred from samples (Fig. 1).

Historically, detrital zircon sample sizes have been chosen based on the probability of detecting the presence or absence of particular zircon age groups (Dodson et al., 1988; Vermeesch, 2004, suggest sample sizes of 60 and 117, respectively). Unfortunately, these sample sizes have been found to be inadequate for representing the relative proportions of age groups in the parent population (Andersen, 2005). Recent analytical advances facilitate the acquisition of larger samples $(n=300-4000)$ that more fully represent the underlying parent population (Fedo et al., 2003; Gehrels et al., 2008; Pullen et al., 2014), but a significant portion of published samples, even in recent studies, are characterized by smaller sample sizes $(n \sim 80-120$; Sharman et al., 2018).

The dominant tool for detrital zircon interpretation has traditionally been visual comparison of probability density plots (Hurford et al., 1984) or age histograms. With the widespread recognition that visual inspection is prone to analyst bias (e.g., Sircombe, 2000; Satkoski et al., 2013), several workers 


\section{Parent population} millions of zircons

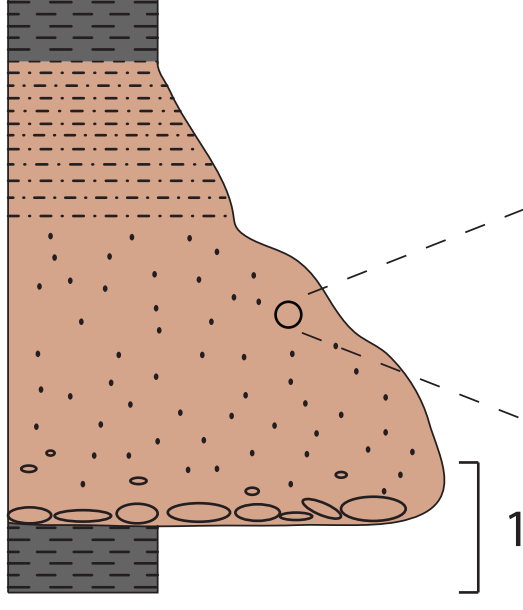

\section{0s - 100s of zircons}

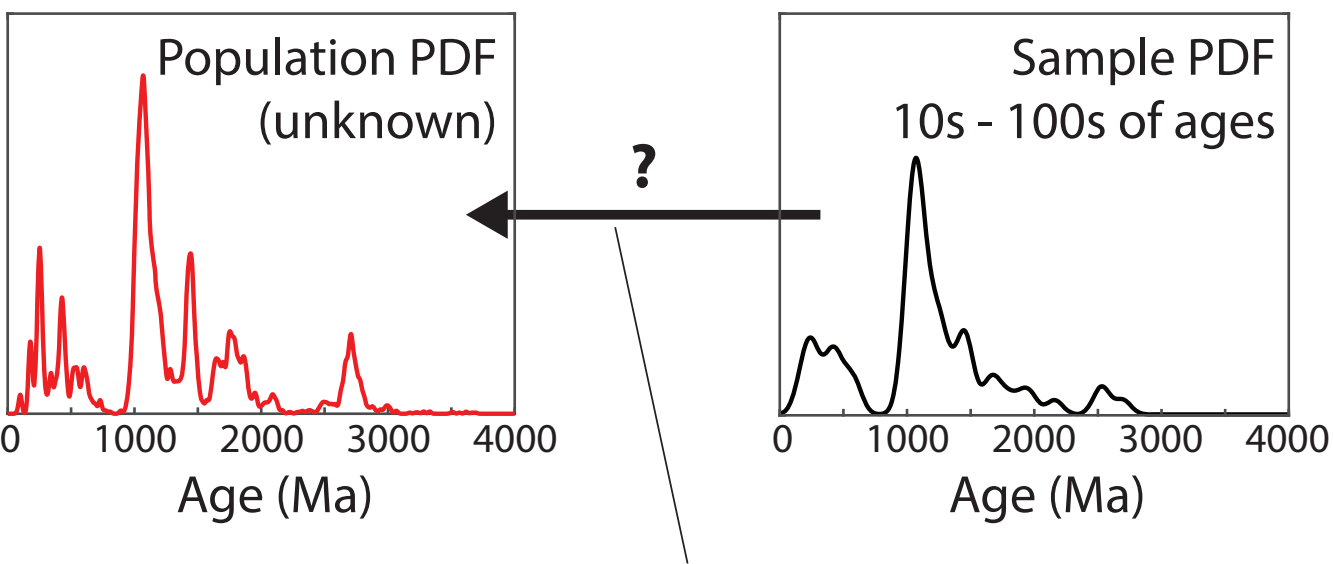

How accurately can we infer the population from the sample?

Figure 1: Detrital zircon populations are shaped by geologic processes and detrital zircon samples are influenced both by these geologic processes and by sampling effects. A sample age distribution can deviate significantly from its parent population distribution, though it is the unknown parent population that is relevant for geological interpretation. Thus, the choice of how to infer parent population characteristics from samples is critical. The population shown is a large detrital zircon dataset (Pullen et al., 2014) and the sample is a random subsample $(n=60)$ of the dataset. Plots use the kernel density estimation method of Botev et al. (2010). 
have proposed quantitative metrics for assessing the correspondence between detrital zircon samples (e.g., Gehrels, 2000; DeGraaff-Surpless et al., 2003; Saylor et al., 2012, 2013; Satkoski et al., 2013; Vermeesch, 2013). The introduction of such quantitative metrics has greatly enhanced the interpretation of detrital zircon age data, facilitating comparison of greater numbers of samples.

Many metrics are used, including quantities associated with the KolmogorovSmirnov (K-S) and Kuiper statistical tests (DeGraaff-Surpless et al., 2003; Lawrence et al., 2011; Vermeesch, 2013; Saylor and Sundell, 2016), and several metrics developed specifically for detrital zircon age distributions, including Similarity (Gehrels, 2000), Cross Correlation (Saylor et al., 2012, 2013) and Likeness (Satkoski et al., 2013). Quantitative metrics of correspondence also permit the application of tools such as multi-dimensional scaling (Vermeesch, 2013; Spencer and Kirkland, 2016) and mixture modeling (e.g., Amidon et al., 2005; Kimbrough et al., 2015; Sharman and Johnstone, 2017; Sundell and Saylor, 2017) to detrital zircon datasets. However, geological interpretations made from these quantitative metrics are limited by the degree to which detrital zircon samples accurately represent sampled parent populations, and there is reason to believe many samples may not be very representative (e.g., Andersen, 2005; Pullen et al., 2014; Ibañez-Mejia et al., 2018). This limitation of existing quantitative metrics is evident in the sample size biasing observed in metric values (Satkoski et al., 2013; Saylor and Sundell, 2016). Relatedly, existing metrics do not provide ways to estimate confidence intervals on metric values, and metric behavior is not well understood beyond indicating that some sample pairs are relatively more alike or less alike than other pairs. Given the complexity of detrital zircon age distributions and the limited sampling that characterizes many datasets, there is an ongoing need for new metrics of correspondence that behave in stable and predictable ways and permit robust estimation of metric uncertainty.

Here, we introduce a new method of inferring and comparing zircon age population distributions that formally incorporates the uncertainty inherent in inferring population distributions from detrital zircon samples. Accounting for this uncertainty is important for detrital zircon studies because most parent populations are too complex to be adequately represented by typical sample sizes, and such accounting may also result in a more stable and predictable correspondence metric. Our method infers sets of potential parent populations that are likely to have produced a given sample, which we refer to as probability model ensembles (PMEs; Fig. 2). We use 
a Bayesian framework for this inference because such a framework allows the rigorous quantification of how well any candidate parent population is statistically supported by a given sample. Within this framework, a Markov Chain Monte Carlo (MCMC) procedure is used to aggregate the potential parent populations contained in each PME. PMEs can be plotted to visually assess the level of constraint that a given zircon age sample places on its parent population. PMEs also form the basis for a new correspondence metric, Bayesian Population Correlation (BPC), which reflects the likelihood that two samples were drawn from the same parent population, as opposed to two distinct parent populations. BPC is the first detrital zircon correspondence metric to display near-complete freedom from sample size bias. In addition, BPC uncertainties can be directly estimated for a specific dataset comparison and BPC results can be predicted from population characteristics using an analytical expression we derive from probability theory. Such predictability permits quantitative interpretations about processes affecting parent populations (e.g., dilution in a sedimentary system). In order to facilitate the use of our methods, we provide MATLABR scripts (also available as a standalone application) for inferring PMEs and calculating BPC (https://github.com/alextye/BPC).

\section{A Bayesian method for inferring ensembles of detrital zircon age populations}

Earth processes act on populations of detrital zircons, of which measured age samples are subsets (Fig. 1). Therefore, making geological interpretations from detrital zircon age data requires using age samples to infer the characteristics of parent population age distributions. Such inference is uncertain because many different parent population age distributions may have produced a given sample set. However, some potential parent populations are far more likely than others. Identifying the set of potential parent populations that are likely to have produced a sample set of observed ages could thus permit more robust geological interpretations of detrital zircon age samples. Here, we present a Bayesian approach for inferring sets of detrital zircon parent populations that are consistent with a set of observed ages. This approach is quantitative and internally consistent, and may lead to more robust interpretation of detrital zircon age samples.

Our method uses Bayes' Theorem to quantify the level of statistical support that a set of zircon ages provides for a candidate parent population. 

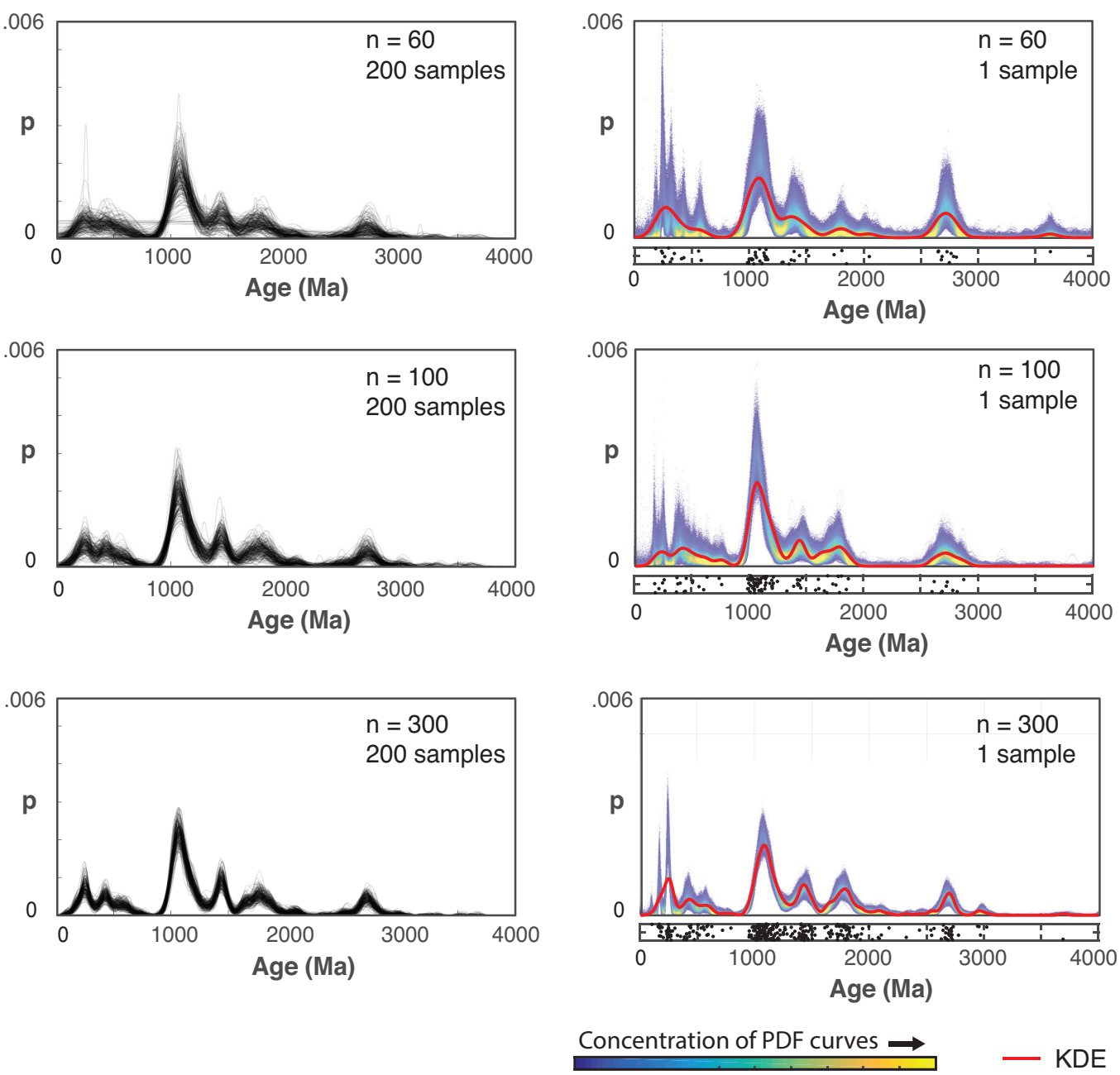

Figure 2: The variability of random samples drawn from a population is accurately captured by Probability Model Ensembles (PMEs) inferred from a single sample. (a) Kernel density estimate curves (KDEs, inferred using the method of Botev et al., 2010) of random samples $(n=60,100,300)$ of a population. Samples were drawn directly from the KDE inferred for the complete dataset of Pullen et al. (2014). (b) Probability model ensembles (PMEs) inferred for a single sample of varying sample size show an excellent match to the values and variability observed in the subsample KDEs. The KDE curve calculated for each sample is also shown in red, indicating that PMEs capture all major age peaks captured by the KDE. Dot plots underneath each panel show the ages of the single random subsample used to infer each PME (vertical scatter used for visual clarity). 
Bayes' Theorem expresses the probability that a particular model, defined by parameters $\boldsymbol{\theta}$, fits a dataset $\boldsymbol{d}$ (Raftery, 1995):

$$
\mathrm{P}(\boldsymbol{\theta} \mid \boldsymbol{d}) \propto \mathrm{P}(\boldsymbol{d} \mid \boldsymbol{\theta}) \mathrm{P}(\boldsymbol{\theta})
$$

with bold symbols indicating vectors. $\mathrm{P}(\boldsymbol{\theta} \mid \boldsymbol{d})$ is the posterior probability that model $\boldsymbol{\theta}$ accurately describes the process that produced dataset $\boldsymbol{d}, \mathrm{P}(\boldsymbol{d} \mid \boldsymbol{\theta})$ is the likelihood of observing data $\boldsymbol{d}$ given probability model $\boldsymbol{\theta}$, and $\mathrm{P}(\boldsymbol{\theta})$ is the prior probability of $\boldsymbol{\theta}$ based on a priori assumptions about the distribution of the model parameters (see Section 2.3). For a given dataset $\boldsymbol{d}, \mathrm{P}(\boldsymbol{\theta} \mid \boldsymbol{d})$, $\mathrm{P}(\boldsymbol{d} \mid \boldsymbol{\theta})$, and $\mathrm{P}(\boldsymbol{\theta})$ are distributions of probability over all possible model parameters $\boldsymbol{\theta}$. The posterior distribution $\mathrm{P}(\boldsymbol{\theta} \mid \boldsymbol{d})$ is particularly useful because it quantifies the support that the data provide for various models as parameters vary. Thus, the distribution of $\mathrm{P}(\boldsymbol{\theta} \mid \boldsymbol{d})$ indicates how tightly or loosely the data constrain each model parameter $\theta_{k}$, whether the parameters covary, etc. In the detrital zircon application, $\boldsymbol{d}$ is the set of ages and analytical uncertainties of one detrital zircon sample and each model is a potential parent population of the observed sample, defined by parameters $\boldsymbol{\theta}$. Sampling the posterior distribution $\mathrm{P}(\boldsymbol{\theta} \mid \boldsymbol{d})$ yields a representative set of the potential parent populations likely to have produced the observed sample, which we call a Probability Model Ensemble (PME). Some detrital zircon samples may support a wide variety of candidate population probability models, whereas others support a more constrained set of models (Fig. 2). Permissible model variability is reflected in the distribution of inferred model parameters in a PME. This information is lost when only a single kernel density estimator (KDE) curve or probability density plot is used for a given detrital zircon sample.

\subsection{Representation of detrital zircon age distributions using basis splines}

Inferring ensembles of potential parent populations in a Bayesian framework requires an efficient method of representing probability density functions (PDFs) using a set of model parameters $\boldsymbol{\theta}$. Efficient representation is provided by basis-spline or b-spline functions (Fig. 3). In b-splining, model curves are generated by summing a series of basis functions $b_{1} \ldots b_{n}$. Each basis function $b_{k}$ is a piecewise function with non-zero value over a limited portion of the $\mathrm{x}$ axis (Fig. 3a), and each basis function has a coefficient $\theta_{k}$ that controls its height. The piecewise function boundaries are called knots, and the shape of each basis function depends on the number of model parameters compared to the number of knots (De Boor, 1978). To generate a 

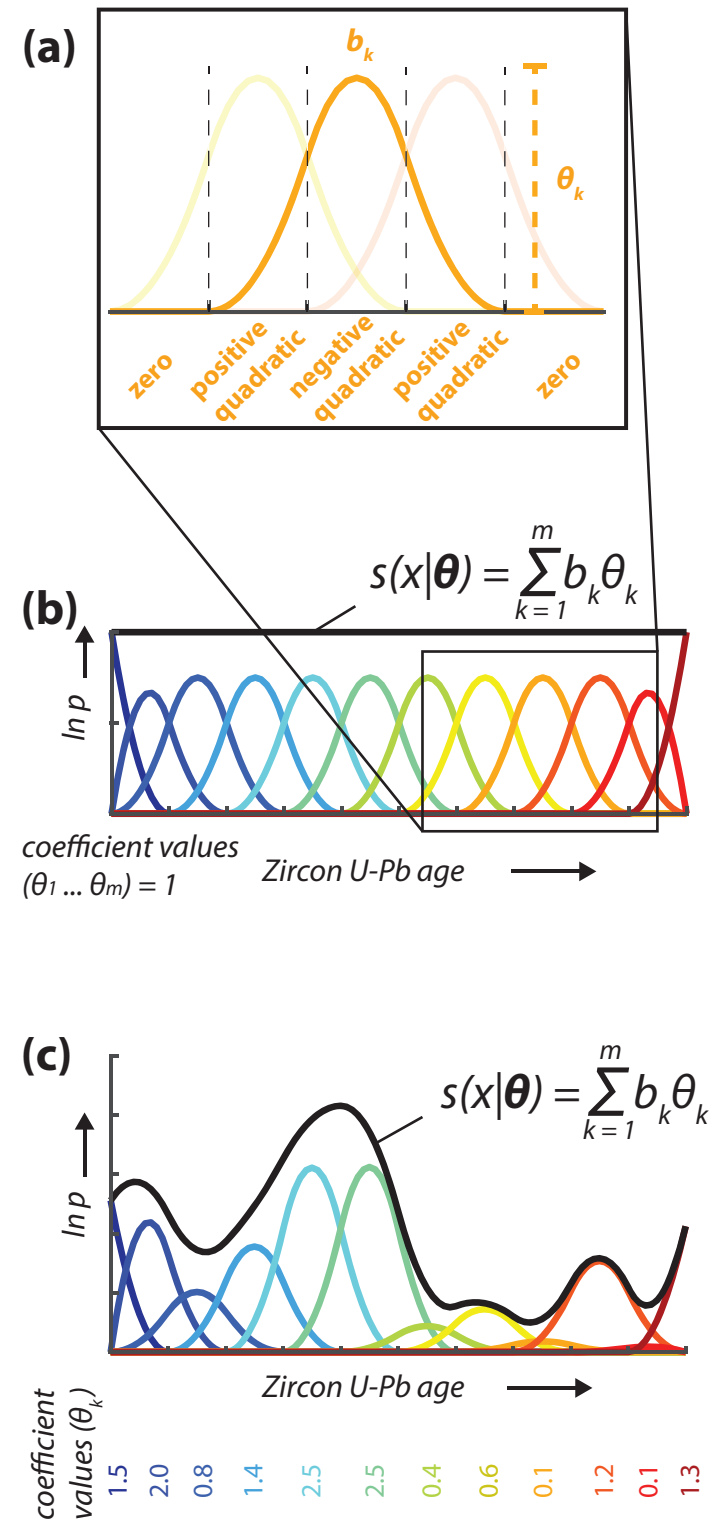

Figure 3: Third order basis splines provide an efficient way to represent probability density functions (PDFs) using a finite number of model parameters. (a) Each spline basis function $b_{k}$ is a piecewise function (boundaries, called knots, shown by dashed lines) that is composed of quadratic pieces and is smooth and differentiable (De Boor, 1978). Importantly, each basis function is defined such that it has non-zero value over only a limited region. A coefficient $\theta_{k}$ is multiplied by the basis function to control its height. (b) In our application, third order basis splines, shown in color, are distributed at regular intervals along the $\mathrm{x}$ axis, which corresponds to zircon $\mathrm{U}-\mathrm{Pb}$ age. The resulting modeled curve, $\mathrm{s}(\mathrm{x})$, is the sum of all basis functions multiplied by their respective coefficients. This example shows the effect of having the coefficient for each basis function equal to one. (c) This

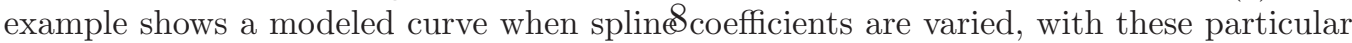
values generated randomly and shown beneath the basis functions. To simplify computation, our application uses these modeled curves as natural log-transformed probability density functions, as discussed in Sections 2.1, S1. 
model curve $s(x \mid \boldsymbol{\theta})$, the basis functions are multiplied by their respective coefficients and summed (Fig. 3b, c). In our method, many basis functions are distributed at fixed, regular intervals over an $\mathrm{x}$ axis that corresponds to zircon $\mathrm{U}-\mathrm{Pb}$ age and basis functions correspond candidate parent population PDFs for a given sample (similar to Eilers and Marx, 1996). In order to simplify our modeling, we use splining to model natural log-transformed probabilities, such that each spline curve $s(x \mid \boldsymbol{\theta})$ is a natural log-transformed probability model (see discussion of the advantages of our approach in Section S4). Each spline curve $s(x \mid \boldsymbol{\theta})$ corresponds with a PDF that we define

$$
g(x \mid \boldsymbol{\theta})=\exp [s(x \mid \boldsymbol{\theta})]
$$

Each candidate parent population PDF, $g(x \mid \boldsymbol{\theta})$, is uniquely identified by its set of basis function coefficients, $\boldsymbol{\theta}$. Sets of likely parent populations aggregated by our method will indicate the range of permissible basis function coefficients warranted by a given zircon age dataset, providing a direct estimate of the uncertainty of age peak heights.

The fact that each basis function has non-zero value over a fixed and localized area means that each parameter $\theta_{k}$ has highly localized influence. The localized influence of each $\theta_{k}$ means that the probability of observing a grain of a certain age is a function of a small subset of the model parameters, greatly simplifying the response of model likelihood $\mathrm{P}(\boldsymbol{d} \mid \boldsymbol{\theta})$ to changes in each model parameter $\theta_{k}$ (see Section S2 for further discussion). Note that the probability model curves $g(x \mid \boldsymbol{\theta})$ generated by this b-spline method could potentially have integrated areas that diverge from unity, meaning they are not true PDFs, and we correct for this divergence in the calculation of likelihood $\mathrm{P}(\boldsymbol{d} \mid \boldsymbol{\theta})$ below. Prior to being plotted or returned to the user, probability model curves $g(x \mid \boldsymbol{\theta})$ are normalized to integrate to unity for ease of use.

The specifics of our implementation of this spline method are chosen to simplify computation and make efficient use of limited computational resources. Our implementation uses 100 spline basis functions, distributed in a mixed log and linear scheme over zircon U-Pb age space (see further discussion in Section S1). Our use of a mixed linear and log basis function arrangement is motivated by the systematics of the U-Pb system and the mass ratios measured to calculate U-Pb ages (see Gehrels, 2000; Gehrels et al., 2008, for further discussion). For ages $<1 \mathrm{Ga}$, the ${ }^{238} \mathrm{U} /{ }^{206} \mathrm{~Pb}$ ratio generally yields the most precise age, and the analytical uncertainty of these ages increases proportional to the measured age (Gehrels, 2000). The pro- 


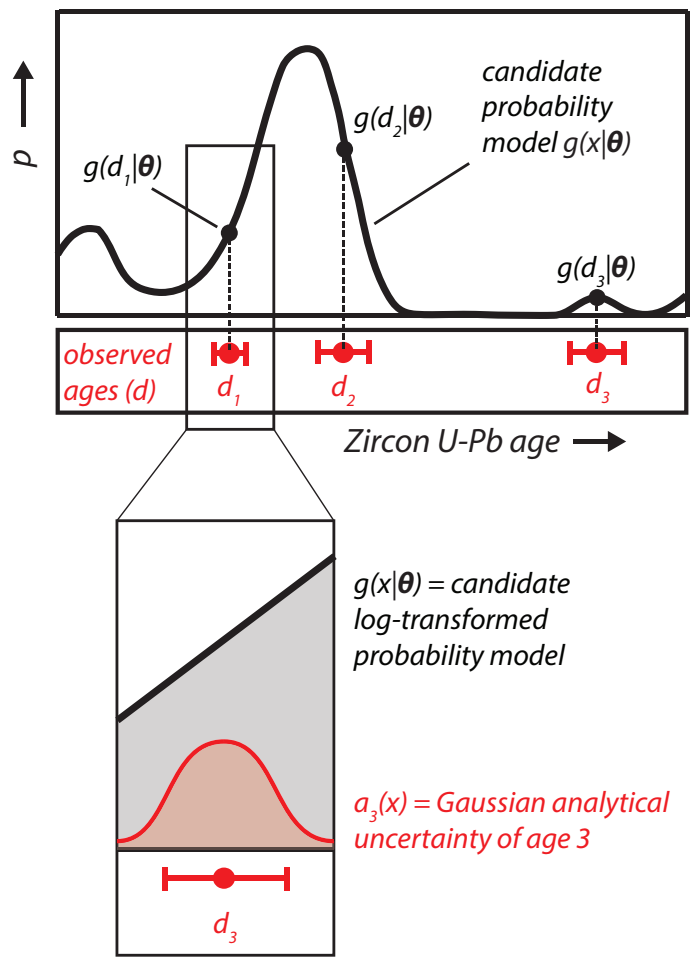

Figure 4: Likelihood $\mathrm{P}\left(d_{i} \mid \boldsymbol{\theta}\right)$ is calculated for a candidate parent population model $g(x \mid \boldsymbol{\theta})$ from the function values of $g(x \mid \boldsymbol{\theta})$ corresponding to observed zircon U-Pb ages in a sample. Likelihoods are calculated by marginalizing (integrating) over the analytical uncertainty of each observed grain age. See discussion in Section 2.2 for more detail.

portionality of analytical uncertainty to age in the $<1$ Ga range suggests that a logarithmic age scale is appropriate in order to capture more precise age peaks at younger ages. For ages $>1 \mathrm{Ga}$, the ${ }^{207} \mathrm{~Pb} /{ }^{206} \mathrm{~Pb}$ ratio generally yields the most precise ages, and analytical uncertainties associated with these ages show an extremely slight, poor negative correlation with age (Gehrels, 2000), such that they are effectively uncorrelated. Because of the effective lack of correlation between ages and uncertainties for ages $>1 \mathrm{Ga}$, basis functions are deployed on a linear age scale at ages $>1 \mathrm{Ga}$, such that basis function width does not change with age. For further discussion of our choice of age scale, see Sections 4.4 and S1. 


\subsection{Quantifying the likelihood $\mathrm{P}(\boldsymbol{d} \mid \boldsymbol{\theta})$}

Likelihood indicates the probability of drawing the observed sample from a candidate parent population. The likelihood of a single age, $\mathrm{P}\left(d_{i} \mid \boldsymbol{\theta}\right)$ is determined by evaluating the PDF that corresponds to the coefficients $\boldsymbol{\theta}$ for datapoint $d_{i}$ (Fig. 4). As mentioned above, we use spline curves to represent natural log-transformed probability density functions, which simplifies our calculations (see further discussion in Section S1). Thus, each spline curve must be exponentiated to evaluate likelihood. As mentioned above, the curves $g(x \mid \boldsymbol{\theta})$ that correspond to model parameters $\boldsymbol{\theta}$ may not integrate to 1 (a requirement for a true PDF), so we normalize the likelihood we infer from these curves by the integrated area of the curve. We account for the analytical uncertainties in each measured age by marginalization: rather than evaluate $g(x \mid \boldsymbol{\theta})$ at a single $x$ value for each datapoint $d_{i}$, we evaluate the surrounding area and weigh the function values $g(x \mid \boldsymbol{\theta})$ by the Gaussian distribution that describes the analytical uncertainty of $d_{i}$ (Fig. 4):

$$
\mathrm{P}\left(d_{i} \mid \boldsymbol{\theta}\right)=\frac{1}{\text { Area } g(x \mid \boldsymbol{\theta})} \int g(x \mid \boldsymbol{\theta}) \cdot a_{i}(x) d x
$$

where $g(x \mid \boldsymbol{\theta})$ is the spline function that corresponds to model parameters $\boldsymbol{\theta}$, and $a_{i}(x)$ is a Gaussian distribution representing the age and analytical uncertainty of $d_{i}$. In our implementation, the integration in Eqn. (3) is solved numerically using Riemann summation, and the area under $g(x \mid \boldsymbol{\theta})$ is also calculated by Riemann summation.

Because each zircon age measurement represents an independent draw from its parent population, the likelihood of observing an entire sample $\boldsymbol{d}$ given model $\boldsymbol{\theta}$ is the product of the likelihood of each individual sample age:

$$
\mathrm{P}(\boldsymbol{d} \mid \boldsymbol{\theta})=\prod_{i=1}^{n} P\left(d_{i} \mid \boldsymbol{\theta}\right)
$$

where $n$ is the sample size.

\subsection{Prior assumptions $\mathrm{P}(\boldsymbol{\theta})$}

Bayesian methods require the explicit statement of prior assumptions, in the form of the probability distribution $\mathrm{P}(\boldsymbol{\theta})$ incorporated into Bayes' Theorem (Eqn. 1). Though it may seem generally desirable to make no assumptions, all methods of estimating population probability distributions from finite samples rely on a set of assumptions. Our method uses the prior 
to enforce the assumption that a distribution should be smooth and uniform in the absence of evidence to the contrary, with peak heights that scale appropriately given the sample size. Such an assumption is an integral part of previous methods to estimate PDFs from finite samples, including kernel density estimation (Silverman, 1986; Botev et al., 2010; Shimazaki and Shinomoto, 2010), as well as previous use of b-splines for density estimation (Eilers and Marx, 1996). We tested three prior distributions to enforce this assumption, including multivariate Gaussian, multivariate Cauchy (Ferguson, 1962), and multivariate Student t distributions. Ultimately, we decided to use the multivariate Student $t$ distribution because of its ease of use and the reasonable behavior of resulting PDFs, as discussed below.

Our first prior assumption is that in the absence of data, any zircon age is as likely as any other within the modeled domain. This assumption is quantified by treating the expected value of each model parameter $\theta_{k}$ as the height of a uniform probability distribution over the domain of $x$ :

$$
E\left(\theta_{k}\right)=\frac{1}{\text { range }(\text { Zircon U-Pb age })}
$$

where range(Zircon $\mathrm{U}-\mathrm{Pb}$ age) here refers to the log-age range over which modeling is conducted, 1 - $4000 \mathrm{Ma}$ in our application. The expected value shown here will yield a uniform distribution over $x$ that integrates to 1 . This expected value is the peak of the prior distribution for each parameter.

The second assumption we can reasonably make about a PDF inferred from a finite sample is that if the sample size increases, the height of the age peaks should increase reflecting the increased confidence gained from a larger sample. To form a rough guideline for how much peak height should increase with added sampling, we use the example of a unimodal age distribution. If a unimodal age distribution contains $n$ ages, then the total integrated probability mass away from the lone age peak should equal $\sim \frac{1}{2 n}$. This is so because if a second age peak existed in the sampled population and constituted a share of the population of $>\frac{1}{2 n}$, then a sample of size $n$ is more likely than not to include at least one zircon age from this second age peak. Conversely, a sample of size $n$ does not provide the statistical power to determine the existence and relative height of age peaks that constitute a share of $<\frac{1}{2 n}$ of the population because those peaks are not likely to be included in a sample of size $n$. Thus, $\sim \frac{1}{2 n}$ is a conservative rough estimate of the total probability mass in a given distribution that is not assigned to recognized age peaks in the distribution. The heavy tailed prior distribu- 
tions that we tested, the Cauchy and multivariate Student t distributions, were able to achieve this desired scaling, whereas the multivariate Gaussian distribution was not. A more detailed and rigorous discussion of this scaling can be found in Section S3.

Our third consideration for the choice of prior distribution is the ability to choose the degree to which a distribution is smoothed, or equivalently stated, the covariance between adjacent basis functions. The multivariate Student t distribution incorporates a covariance matrix, which makes it easy to specify the covariance between adjacent basis functions, while the multivariate Cauchy distribution does not.

The multivariate Student t prior enforces norms that are common to all methods of inferring continuous PDFs from finite datasets of which we are aware, including those that are commonly used with detrital zircon $\mathrm{U}-\mathrm{Pb}$ data (e.g., Botev et al., 2010). We parameterize the multivariate Student $\mathrm{t}$ distribution using the expected parameter values described by Eqn. (5). The covariance matrix we use to parameterize the Student $t$ distribution defines the variability of each basis function coefficient as well as the covariance between the coefficients. Coefficient variance is defined by the diagonal elements of the covariance matrix. Coefficient covariance, which controls the smoothness of modeled PDFs, is defined by the off-diagonal elements of the covariance matrix. The covariance matrix values we use to achieve the desired behavior discussed above are diagonal element values of $13.5^{2}$ and off-diagonal element values that decay away from the diagonal, following the expression $13.5^{2} e^{\frac{-|i-j|}{100}}$, where $i$ and $j$ are the row and column index of the respective element. The distribution has 5 degrees of freedom (Lange et al., 1989). Despite the performance differences between the multivariate Student t distribution and multivariate Gaussian described here, we found that calculated BPC values (discussed below) were minimally affected by the choice of whether to use a multivariate Gaussian or multivariate Student t prior distribution.

\subsection{Aggregation of probability model ensembles (PMEs)}

Representative PMEs are aggregated for a given sample using a Markov Chain Monte Carlo (MCMC) method, with the Metropolis-Hastings algorithm used to determine whether a potential parent population is added to the PME. In an MCMC method, the unknown posterior distribution $\mathrm{P}(\boldsymbol{\theta} \mid \boldsymbol{d})$ is explored by a random walker moving through an $m$-dimensional space, where $m$ is the number of model parameters and each dimension corresponds 
to a single parameter. In our case, the movement of the walker and the resulting Markov chain (the record of the walker's path through the space) are governed by the Metropolis-Hastings algorithm, resulting in a Markov chain that is a representative sample of the posterior distribution $\mathrm{P}(\boldsymbol{\theta} \mid \boldsymbol{d})$ (Gelman et al., 2013). In our implementation, the random walker begins at the maximum likelihood model (found by inversion; Shanno, 1970) so the walker wastes no steps in reaching the region of high posterior probability. In order to ensure adequate sampling of the posterior distribution $\mathrm{P}(\boldsymbol{\theta} \mid \boldsymbol{d})$, we require the random walker to run for a greater number of steps if parameter values are highly autocorrelated from one step to the next (Geyer, 1992). MCMC convergence is discussed further in Section S2. The typical number of steps in the Markov chain is $10^{4}$ to $10^{5}$.

Because the Metropolis-Hastings method generates representative samples of the posterior $\mathrm{P}(\boldsymbol{\theta} \mid \boldsymbol{d})$, models $\boldsymbol{\theta}$ are represented in a PME to the degree that they are supported by the data. Thus, the permissible variability in models (in this case, parent population PDFs) is represented in this sample of the posterior. Posterior distributions become more tightly clustered the greater the level of constraint the data provide on the model parameters. For instance, when the size of a detrital zircon $\mathrm{U}-\mathrm{Pb}$ age sample increases, the permissible variability in potential parent populations shrinks (Fig. 2).

\section{Estimating correspondence between PMEs: Bayesian Popula- tion Correlation (BPC)}

In order to assess the correspondence of two zircon age populations using the robust constraints contained in PMEs, we develop a new comparative metric called Bayesian Population Correlation (BPC). BPC incorporates the uncertainties in parent population inference that are reflected in PMEs. Specifically, BPC compares the probabilistic support for two alternative hypotheses: the joint hypothesis $\left(H_{J}\right)$, in which two observed samples are from one joint parent population, and the separate hypothesis $\left(H_{S}\right)$, in which each sample is drawn from its own, separate parent population. BPC depends on the relative likelihood of the two hypotheses, $H_{J}$ and $H_{S}$, which can vary over many orders of magnitude, so we define the relative likelihood magnitude $\Lambda$ as the natural $\log$ of the relative likelihood of $H_{J}$ versus $H_{S}$ : 


$$
\begin{aligned}
\Lambda & =\ln \left[\frac{\mathrm{P}\left(H_{J}\right)}{\mathrm{P}\left(H_{S}\right)}\right]=\left\langle\ln \left[\frac{\mathrm{P}\left(\boldsymbol{d}_{J} \mid\left\{\boldsymbol{\theta}_{J}\right\}\right)}{\mathrm{P}\left(\boldsymbol{d}_{1} \mid\left\{\boldsymbol{\theta}_{1}\right\}\right) \mathrm{P}\left(\boldsymbol{d}_{2} \mid\left\{\boldsymbol{\theta}_{2}\right\}\right)}\right]\right\rangle \\
& =\left\langle\ln \mathrm{P}\left(\boldsymbol{d}_{J} \mid\left\{\boldsymbol{\theta}_{J}\right\}\right)\right\rangle-\left\langle\ln \mathrm{P}\left(\boldsymbol{d}_{1} \mid\left\{\boldsymbol{\theta}_{1}\right\}\right)\right\rangle-\left\langle\ln \mathrm{P}\left(\boldsymbol{d}_{2} \mid\left\{\boldsymbol{\theta}_{2}\right\}\right)\right\rangle
\end{aligned}
$$

where $\boldsymbol{d}_{1}$ and $\boldsymbol{d}_{2}$ are the age data of sample 1 and sample 2 , and $\boldsymbol{d}_{J}$ is the union of $\boldsymbol{d}_{1}$ and $\boldsymbol{d}_{2}$, representing the combined data of samples 1 and 2. We refer to $\boldsymbol{d}_{J}$ as the hypothetical joint sample. $\left\{\boldsymbol{\theta}_{1}\right\},\left\{\boldsymbol{\theta}_{2}\right\}$, and $\left\{\boldsymbol{\theta}_{J}\right\}$ are the PMEs of samples 1 and 2 and the hypothetical joint sample, inferred using the MCMC method described above. Here and in subsequent expressions, angular brackets indicate the mean ensemble natural log likelihood, which is calculated by taking the mean of natural $\log$ likelihood values of the full PME. In general, $\mathrm{P}\left(\boldsymbol{d}_{J} \mid\left\{\boldsymbol{\theta}_{J}\right\}\right)=\mathrm{P}\left(\boldsymbol{d}_{1} \mid\left\{\boldsymbol{\theta}_{J}\right\}\right) \cdot \mathrm{P}\left(\boldsymbol{d}_{2} \mid\left\{\boldsymbol{\theta}_{J}\right\}\right)$ because $\boldsymbol{d}_{J}$ is composed of $\boldsymbol{d}_{1}$ and $\boldsymbol{d}_{2}$ and zircon ages $d_{i}$ are independent draws from a population so their probabilities are multiplicative. Substituting this result into Eqn. (6) yields

$$
\Lambda=\left\langle\ln \mathrm{P}\left(\boldsymbol{d}_{1} \mid\left\{\boldsymbol{\theta}_{J}\right\}\right)\right\rangle+\left\langle\ln \mathrm{P}\left(\boldsymbol{d}_{2} \mid\left\{\boldsymbol{\theta}_{J}\right\}\right)\right\rangle-\left\langle\ln \mathrm{P}\left(\boldsymbol{d}_{1} \mid\left\{\boldsymbol{\theta}_{1}\right\}\right)\right\rangle-\left\langle\ln \mathrm{P}\left(\boldsymbol{d}_{2} \mid\left\{\boldsymbol{\theta}_{2}\right\}\right)\right\rangle
$$

For ease of interpretation, we seek to scale $\Lambda$ to occupy the range between 0 and 1 . Such scaling can be accomplished by normalizing $\Lambda$ with respect to two end member scenarios: (1) identical parent populations, which will produce the maximum $\Lambda$ value and (2) parent populations with no shared ages, which will produce the minimum $\Lambda$ value. Here, we calculate these end member results analytically, making the simplifying assumption that samples are large enough to accurately represent parent population PDFs (Fig. 5). Following from Eqns. $(6,7)$, the expected value of $\Lambda, \Lambda_{i d e a l}$, is defined as

$$
\Lambda_{\text {ideal }}=\ln \left[\frac{\mathrm{P}\left(H_{J}\right)}{\mathrm{P}\left(H_{S}\right)}\right]=\ln \left[\frac{\mathrm{P}\left(\boldsymbol{d}_{1} \mid F_{J}\right) \mathrm{P}\left(\boldsymbol{d}_{2} \mid F_{J}\right)}{\mathrm{P}\left(\boldsymbol{d}_{1} \mid F_{1}\right) \mathrm{P}\left(\boldsymbol{d}_{2} \mid F_{2}\right)}\right]
$$

where $F_{1}, F_{2}$, and $F_{J}$ are well characterized parent population PDFs and the data are assumed to be independent observations such that $\mathrm{P}\left(\boldsymbol{d}_{J}\right)=$ $\mathrm{P}\left(\boldsymbol{d}_{1}\right) \cdot \mathrm{P}\left(\boldsymbol{d}_{2}\right)$. In the first endmember case, where samples are drawn from identical parent populations, $F_{1}=F_{2}=F_{J}$, so Eqn. (8) shows that $\Lambda_{\text {ideal }}=$ 0 . In the second endmember case, where parent populations are perfectly non-overlapping, the probability mass of $F_{J}$ must be spread out in order to 


\section{End member 1:} perfectly corresponding populations
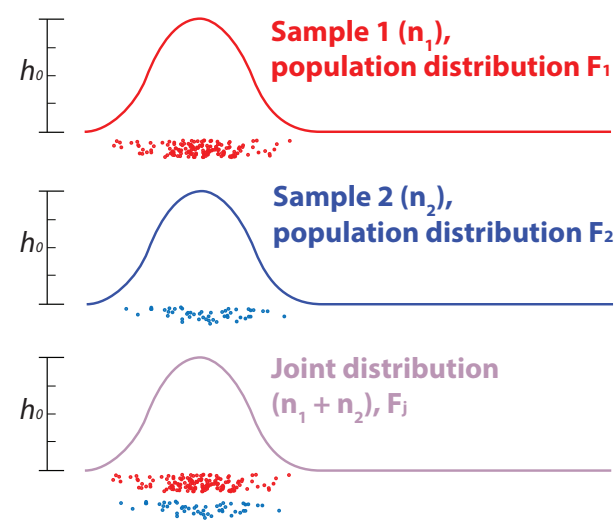

End member 2: perfectly non-overlapping populations
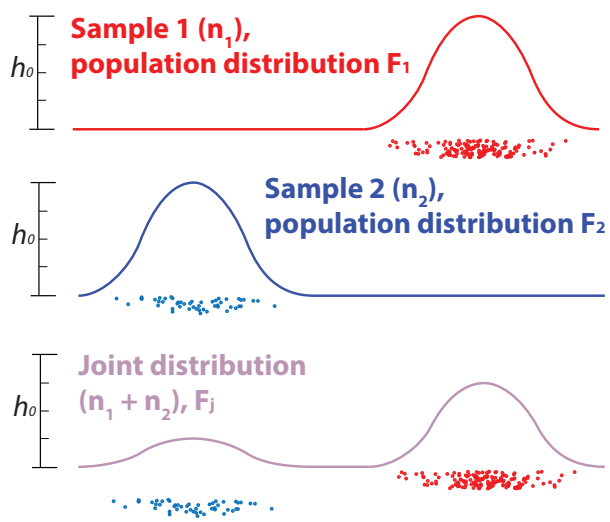

Figure 5: Two idealized end member scenarios (perfectly corresponding populations and perfectly non-overlapping populations) assist with the calculation of upper and lower bounds of the relative likelihood magnitude $\Lambda$. Scale bar shows $h_{0}$, the height of the age peak of the unimodal samples (see Section 3).

accommodate samples from both populations. In this case, $F_{J}$ is the sum of $F_{1}$ and $F_{2}$ each weighted by the proportion of total data that comes from their respective samples:

$$
F_{J}=\frac{n_{1}}{n_{1}+n_{2}} F_{1}+\frac{n_{2}}{n_{1}+n_{2}} F_{2}
$$

where $n_{1}$ and $n_{2}$ are the sample sizes of the two samples. This definition of $F_{J}$ maximizes the likelihood of observing $\boldsymbol{d}_{J}$, which is the union of $\boldsymbol{d}_{1}$ and $\boldsymbol{d}_{2}$. In order to find the minimum $\Lambda$ value using Eqn. (8), we must relate the likelihood of observing a given zircon age $d_{i}$ under $F_{J}, \mathrm{P}\left(d_{i} \mid F_{J}\right)$, to the likelihood of observing the same age under $F_{1}$ and $F_{2}, \mathrm{P}\left(d_{i} \mid F_{1}\right)$ and $\mathrm{P}\left(d_{i} \mid F_{2}\right)$. Eqn. (3) shows that multiplying $F$ by a constant will also scale $\mathrm{P}\left(d_{i} \mid F\right)$ by the same constant. Therefore, $\mathrm{P}\left(d_{i} \mid F_{J}\right)$ can be calculated from $\mathrm{P}\left(d_{i} \mid F_{1}\right)$ and $\mathrm{P}\left(d_{i} \mid F_{2}\right)$ as follows:

$$
\mathrm{P}\left(d_{i} \mid F_{J}\right)=\frac{n_{1}}{n_{1}+n_{2}} \mathrm{P}\left(d_{i} \mid F_{1}\right)+\frac{n_{2}}{n_{1}+n_{2}} \mathrm{P}\left(d_{i} \mid F_{2}\right)
$$

Considering first the case of $d_{i}$ taken from sample 1, we assert that because populations 1 and 2 are perfectly non-overlapping, $\mathrm{P}\left(d_{i, 1} \mid F_{2}\right)=0$, which allows eliminating the second term of Eqn. (10). Because the likelihood of 
observing sample $\boldsymbol{d}_{1}$ is the product of the likelihood of each individual age in the sample $d_{i, 1}$ (Eqn. 4) and because $\mathrm{P}\left(d_{i, 1} \mid F_{2}\right)=0$, it follows from Eqn. (10) that

$$
\mathrm{P}\left(\boldsymbol{d}_{1} \mid F_{J}\right)=\left(\frac{n_{1}}{n_{1}+n_{2}}\right)^{n_{1}} \mathrm{P}\left(\boldsymbol{d}_{1} \mid F_{1}\right)
$$

By the same reasoning presented above, $\mathrm{P}\left(d_{i, 2} \mid F_{1}\right)=0$, so Eqn. (11) applies when the subscripts 1 and 2 are swapped, as well. When this result is substituted into Eqn. (8), numerator and denominator terms cancel, revealing the expression for $\Lambda_{\text {ideal }}$ when there is no population overlap, which we term $\Lambda_{\min }$ :

$$
\Lambda_{\text {min }}=\ln \left[\left(\frac{n_{1}}{n_{1}+n_{2}}\right)^{n_{1}}\left(\frac{n_{2}}{n_{1}+n_{2}}\right)^{n_{2}}\right]
$$

We define Bayesian Population Correlation as a remapping of $\Lambda$ onto the range of 0 to 1 , which we accomplish using the two endmember cases described above:

$$
B P C=1-\left(\frac{\Lambda}{\Lambda_{\min }}\right)
$$

Because $\Lambda_{\min }$ accounts for the sizes of each sample, expected BPC values should remain insensitive to sample size, (though BPC uncertainties vary with sample size, see Section 5). Two samples drawn from identical parent populations should produce a BPC value of 1 , whereas two samples drawn from completely distinct populations should yield a $\mathrm{BPC}$ value of 0 . The variation of this metric between 0 and 1 , with higher values indicating increasingly similar populations, motivates our use of the word 'correlation' in its name. We note that BPC is similar to the Bayes factor of Jeffreys (1998), though the Bayes factor is based on comparison of posterior values rather than likelihood values.

We note that BPC occasionally exceeds 1 for two very similar samples, which can be explained by the fact that both priors and likelihood influence the construction of PMEs, but BPC is based solely on likelihood. Consider the example of a sample being compared against itself, such that to construct the hypothetical joint PME, all observed zircon ages in the sample are doubled. Our priors are tuned such that larger sample sizes will increase age peak heights (see discussion in Section 2.3). Thus, in the case of two identical samples, the joint PME will have taller age peaks than the two identical 
separate PMEs. These taller peaks result in higher likelihoods for each zircon age than the age peaks of the PME inferred for the sample by itself. The higher likelihood of each sample age under the joint PME results in a BPC value slightly greater than 1 (Eqns. 6, 13). This same phenomenon can occur for two nearly identical samples. Despite this undesired behavior, we believe the use of a likelihood ratio is justified because of its well understood behavior. When BPC is greater than 1, estimated uncertainty (see Section 5) typically includes 1.

\section{Testing the behavior of BPC}

We conduct a suite of resampling experiments to test the behavior of BPC. First, a simple experiment involving unimodal populations with variable overlap tests the basic functioning of BPC. Then, the effect of changing sample size on BPC is tested using resampling experiments conducted with published detrital zircon datasets. Finally, we also conduct experiments to assess the effect on BPC of varying the proportion of shared ages in two compared parent populations.

\subsection{BPC behavior in a simple case}

In order to verify that BPC behaves as intended in a simple case, BPC was evaluated for samples of two synthetic, Gaussian parent populations that are systematically displaced relative to one another (Fig. 6). For this experiment, PMEs were modeled over an $x$ value domain of 0 to 6.9 , which corresponds to the natural logarithmic transformation of the age range 1 $1000 \mathrm{Ma}$, thus paralleling our modeling procedure for zircon U-Pb age data $<1 \mathrm{Ga}$. One Gaussian parent is centered on 2.5 and a second Gaussian parent of equal width is defined with its center located at 3, 3.5, 4, etc. The standard deviation of each Gaussian parent population is 0.5. Random values are drawn from these Gaussian parent populations and compared to one another using BPC (Fig. 6). The analytical uncertainty ascribed to each log-age was $\sim 0.01$, which equates to $1 \%$ of its value in linear space. The results of this experiment show that $\mathrm{BPC}$ varies smoothly between 0 and 1 with increasing population overlap independent of sample size, as expected.

\subsection{Independence from sample size bias}

To assess the effects of sample size on BPC for realistic samples, BPC is tested on samples drawn from existing detrital zircon datasets (Pullen 


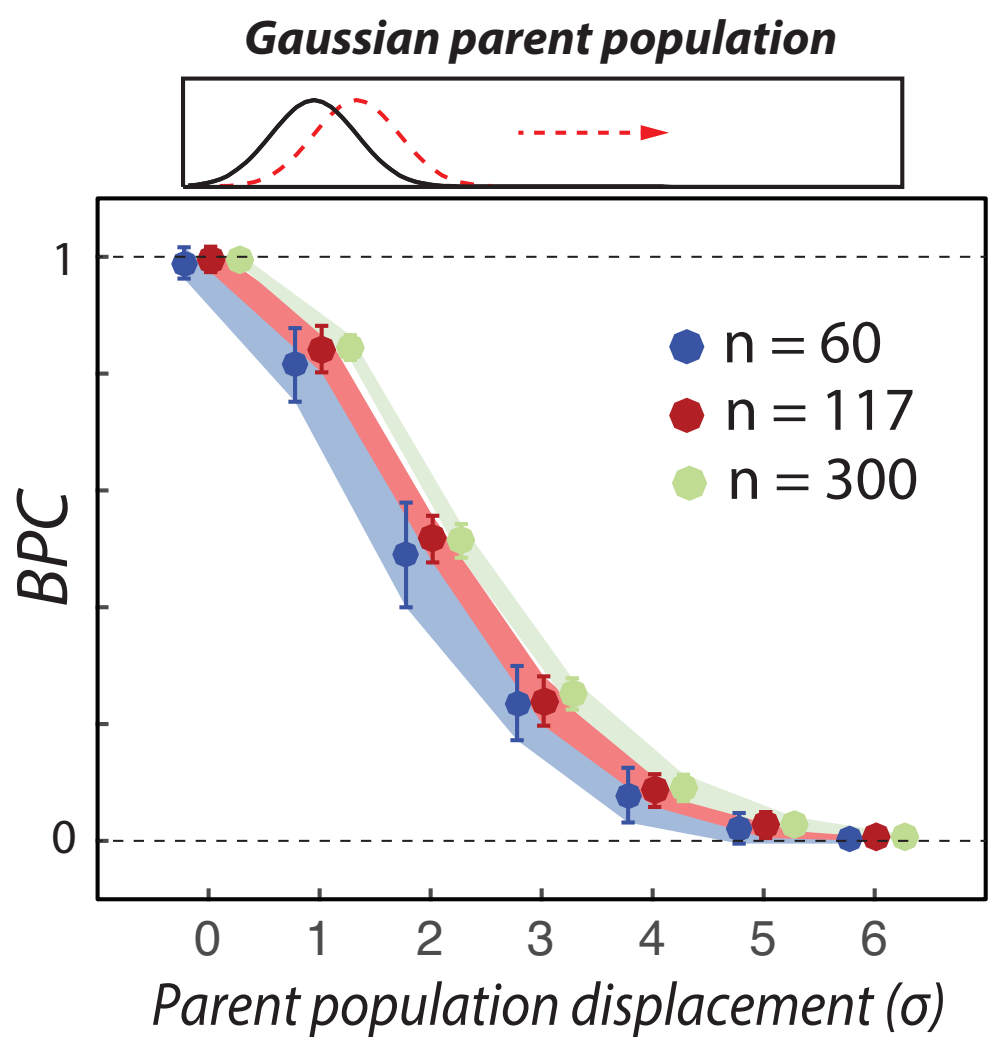

Figure 6: Experiments with a range of partially overlapping Gaussian parent populations show that BPC inhabits the full metric range between 0 and 1 and returns consistent values for all tested sample sizes. Two Gaussian parent populations are systematically displaced from one another (expressed in terms of peak-width units $\sigma$ ) and sampled randomly at sample sizes $n=60,117,300$. Resulting samples are compared using BPC. Each symbol represents the mean and standard deviation of metric values across twenty experiments and symbols are plotted with small horizontal offsets for visual clarity. 
(a)
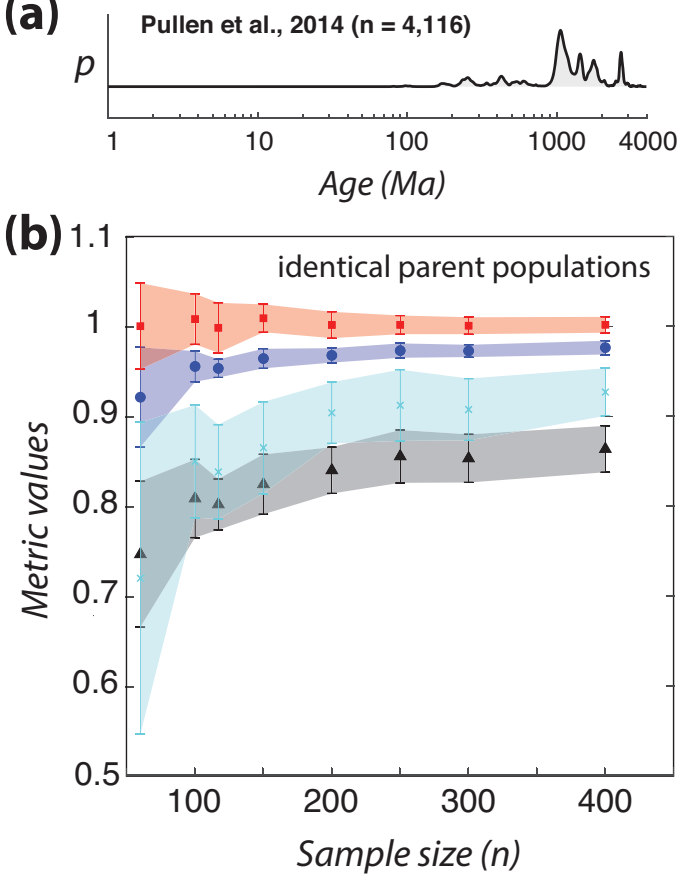

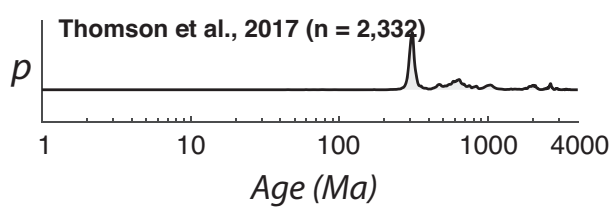

(c) 0.7

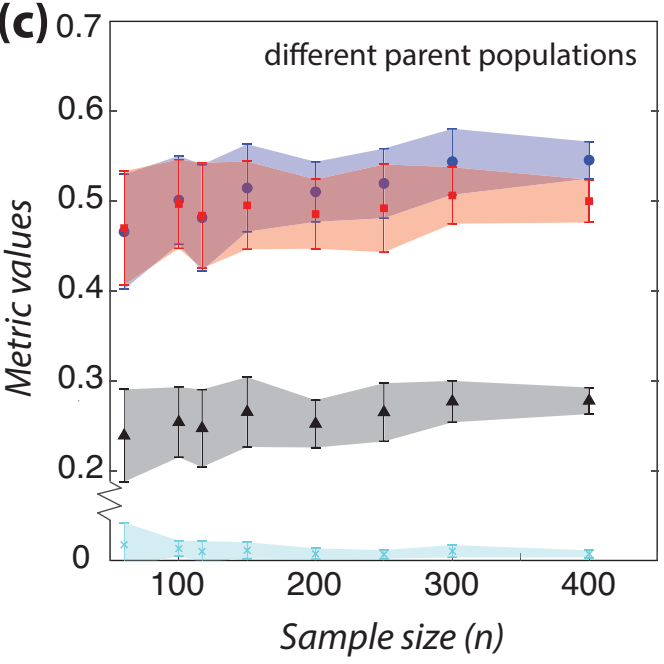

Bayesian Population Correlation (BPC) (this study) Similarity (Gehrels, 2000)

A Likeness (Satkoski et al., 2013) X Cross Correlation (Saylor et al., 2012, 2013)

Figure 7: BPC calculated from random subsamples of two large detrital zircon datasets are unbiased with respect to sample size, in contrast to existing detrital zircon correspondence metrics. (a) Datasets of Pullen et al. (2014); Thomson et al. (2017) were resampled by drawing ages directly from the KDE (Botev et al., 2010) calculated for each dataset. (b) Metric results are shown for when two subsamples of a single population (the dataset of Pullen et al., 2014) are compared. (c) Results are shown for when subsamples are drawn from two different populations (the datasets of Pullen et al., 2014; Thomson et al., 2017). The key shows the plotted metrics, and previously published metrics were calculated using KDE curves estimated using the method of Botev et al. (2010). BPC is not based on a KDE method. In (c), note the broken vertical axis. 
et al., 2014; Thomson et al., 2017) with variable sample size (Fig. 7). The synthetic subsamples used for testing are drawn directly from the KDEs inferred for the large datasets of (Pullen et al., 2014; Thomson et al., 2017), which mirrors the process of sampling a population. Cases of identical parent populations (two samples drawn from the dataset of Pullen et al. (2014)) and different parent populations (one sample drawn from the dataset of Pullen et al. (2014), one sample drawn from the dataset of Thomson et al. (2017)) are both tested, and in each case sample sizes between $n=60$ and $n=400$ are used. BPC values calculated between random subsamples of identical populations cluster around 1, with decreasing scatter as sample size increases (Fig. 7b). When BPC is calculated between random subsamples of two different parent populations, it demonstrates a consistent value across a range of tested sample sizes (Fig. 7c), again with decreasing scatter as sample size increases.

The consistent behavior of $\mathrm{BPC}$ and lack of sample size biasing contrasts with the behavior of published metrics for detrital zircon correspondence (Fig. 7) including Similarity (Gehrels, 2000), Cross Correlation (Saylor et al., 2012, 2013) and Likeness (Satkoski et al., 2013). These published metrics are functions of KDE curves inferred for samples, and the values of these metrics are dependent on both sample size and KDE method chosen (Saylor and Sundell, 2016, review and test these metrics). Both of these dependencies are problematic because they indicate that metric value is not solely a function of differences between detrital zircon populations, and therefore that these metrics do not solely reflect geologic processes. For comparison with BPC, Figure 7 shows values of Similarity, Likeness, and Cross Correlation calculated using the KDE method of Botev et al. (2010), which is widely used and implemented in the DensityPlotter software of Vermeesch (2012). It can be seen that the mean values of Similarity, Likeness, and Cross Correlation are each systematically related to sample size in at least one of the two cases. In addition, of these three metrics, there is no single metric that minimizes sample size bias in the cases of both identical and different parent populations. In the case of identical parent populations, Similarity shows the smallest change in value from $n=60$ to $n=400$, whereas in the case of different parent populations, Similarity shows a greater change in value than Likeness or Cross Correlation. Additional testing using other KDE methods documents changes in metric behavior when different KDE methods are used (see Section S4, Fig. S5). In addition, we also find that the test statistics and $p$-values associated with the Kolmogorov-Smirnov and Kuiper tests, which 
have been used to assess detrital zircon correspondence, are biased according to sample size for our resampling experiments (Fig. S4). The sample size biasing and dependence on KDE method of these previously published metrics is explored in more detail elsewhere (Saylor and Sundell, 2016). BPC, which shows minimal or no sample size biasing and is not based on kernel density estimation, avoids these issues.

\subsection{Interpreting the meaning of $B P C$ values}

Quantitative interpretation of existing metrics is currently limited to determining whether one pair of samples is more or less alike than another pair of samples. For a metric to have direct implications for earth processes, the metric would need to directly constrain parent population characteristics such as the proportion of ages in one population that are shared with another population. If a metric were to display a robust functional relationship between the shared proportions of populations and metric value, then the metric could be used to provide novel quantitative constraints on processes that affect detrital zircon populations. BPC has the potential to display such a functional relationship, in large part due to its grounding in probability theory. We have already demonstrated the use of probability theory to derive accurate expected BPC values for the cases of identical parent populations and parent populations with no shared ages (Section 3). Therefore, it stands to reason that expected BPC values derived analytically in a similar fashion could potentially predict experimental BPC values for partially overlapping parent populations, as well.

We derive an equation for expected BPC value as a function of the proportion of shared ages of two detrital zircon populations (Section S5) and test it against BPC values from resampling experiments (Fig. 8). If these expected values are accurate predictions of BPC values calculated between pairs of detrital zircon samples, then BPC has a functional relationship to the shared proportions of two populations. Such a relationship would permit quantitative interpretations of the effects of earth processes on detrital zircon populations directly from metric value. In order to test whether measured BPC values conform to the predictions derived from probability theory, we devised additional resampling experiments where the proportion of ages shared between two populations is systematically varied (Fig. 8). The dataset of Pullen et al. (2014) is divided into three broad subgroups (young, 0-800 Ma; middle, 800-1550 Ma; and old, 1550-4000 Ma) and synthetic parent populations are constructed using various mixtures of these subgroups. 

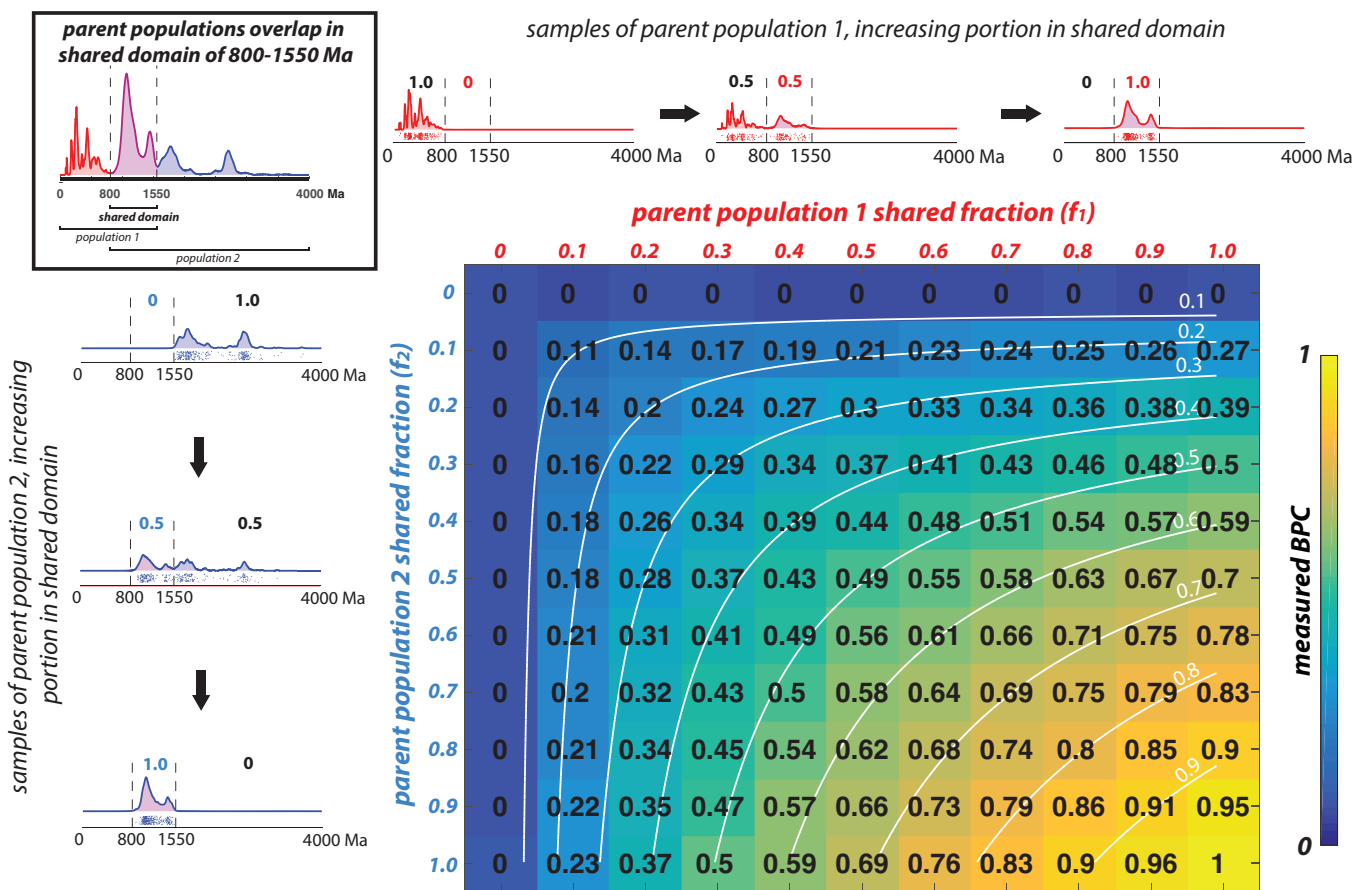

Figure 8: Comparison of numerical experiments to analytical predictions indicates that $\mathrm{BPC}$ is well described as a function of the proportions of zircon age groups that are shared between two samples. To model the effects of partially overlapping zircon age samples, the dataset of Pullen et al. (2014), is divided into three subgroups, 0-800 Ma, 800-1550 Ma, and 1550-4000 Ma (see upper left panel). These subgroups are used to generate synthetic zircon parent populations with variable proportions of shared ages (see description in Section 4). Upper and lefthand margins show typical samples drawn from synthetic populations so generated. The greater the proportion of each synthetic population that lies in the shared subgroup, the greater the expected correspondence. The shared fractions of each synthetic parent population are represented by two independent variables, $f_{1}$ and $f_{2}$, which vary between 0 and 1. $f_{1}$ and $f_{2}$ form the $x$ and $y$ axes of the table (note that $f_{2}$ increases from top to bottom). In the main plot, colors and black numbers correspond to mean BPC values from four experiments for each coordinate pair $\left(f_{1}, f_{2}\right)$. In each experiment, samples of size $n=300$ were drawn from parent populations 1 and 2 . White contours show analytically predicted $\mathrm{BPC}_{\text {ideal }}$ values, given by Eqn. (S10) in Section S5, which show an excellent match with observed results. The uncertainty of each experimental BPC value shown is 0.02 . 
The subgroup boundaries are chosen to fall between coherent age peaks in the data. For each experiment, two synthetic parent populations are defined as follows: parent 1 includes grains of the young and middle subgroups and parent 2 includes grains of the middle and old subgroups. Thus, a portion of each synthetic parent population consists of grains of the shared middle age domain. The proportion of each parent population that belongs to the shared domain is independently varied from 0 (no middle aged grains) to 1 (entirely middle aged grains) and these proportions are represented as $f_{1}$ and $f_{2}$. The synthetic parent populations are then sampled and BPC is calculated; this procedure is repeated 4 times for each coordinate pair $\left(f_{1}, f_{2}\right)$ with sample size $n=300$ and results are shown in black in Figure 8.

The results of these experimental results show that BPC varies smoothly between zero and one as a function of the shared proportions of detrital zircon populations. BPC values of zero indicate no shared ages between two detrital zircon populations, whereas values of 1 indicate that the samples are likely to have been drawn from identical parent populations. BPC values between 0 and 1 indicate partial overlap of the ages contained in the two populations. We also compare the results of these numerical experiments to expected BPC values derived analytically for variable $f_{1}$ and $f_{2}$ (see Section S5, Eqn. S10). Expected BPC values are shown in white contours in Figure 8. BPC results from the resampling experiments conform almost perfectly to the expected values, indicating that BPC values can be accurately predicted from probability theory.

In order to ensure this correspondence between measured and expected $\mathrm{BPC}$ values is robust under a variety of realistic conditions, we performed two additional variants of the experiment described above. First, we divide the Pullen et al. (2014) dataset into 20 natural age peaks and then assign each of those age peaks to one of three subgroups such that the age peaks of each subgroup are interspersed across the full range of ages present in the dataset. Random subsamples are drawn with replacement from the subgroups and BPC calculated as above. The results of this experimental scheme are indistinguishable from the results when the three subgroups are defined as 0-800 Ma, 800-1550 Ma, and 1550-4000 Ma (Fig. 8). Thus, BPC values can be accurately predicted even when the shared and unshared age peaks of two populations are dispersed throughout the range of age values present. In order to test the effect of varying sample size on the predictability of BPC values, the sizes of compared samples were systematically varied for selected combinations of $\left(f_{1}, f_{2}\right)$. BPC values were within uncertainty of expected 
values (see below for estimation of BPC uncertainties) for all tested cases, including when sample sizes differ by up to an order of magnitude. The results of these additional experiments show that $\mathrm{BPC}$ values can be accurately predicted from $\left(f_{1}, f_{2}\right)$ over a variety of sample sizes and in situations where shared and unshared age peaks are interspersed in compared populations.

The functional relationship between BPC and shared population proportions can be inverted, meaning that a BPC value found for two real detrital zircon samples can be used to constrain the shared proportions of the sampled parent populations, which are unknown for natural samples. The shared proportions of two detrital zircon populations $\left(f_{1}, f_{2}\right)$ are directly affected by geologic processes such as sediment mixing (see Niemi, 2013, and Section S5 for further discussion). Thus, the ability to infer the shared proportions of two detrital zircon populations permits quantitative interpretations of geologic processes from detrital zircon age samples that were not previously possible. The near perfect conformity of BPC values to theoretical expectations for partially overlapping parent populations suggests that expected BPC values could be derived for other more complicated scenarios as well, such as multiple partially overlapping age categories.

\subsection{Limitations of our implementation of $B P C$}

The fixed location and width of b-spline basis functions suggests that if age peaks are narrow and closely spaced enough, then these age peaks might not be differentiated in PMEs. To quantitatively assess the effect of resolution issues on PME inference and BPC, we performed repeated experiments using synthetic Gaussian parent populations (similar to Fig. 6) with variable widths. As might be expected, these experiments indicated that our PME inference method cannot resolve age peaks that are narrower than a single spline basis function. If the major differences between two populations are defined by small offsets between such narrow age peaks, calculated BPC values may be too high. Our mixed log and linear age scale (Section 2.1) results in the following minimum widths for age peaks to be fully resolved by our method. Spline basis functions are distributed on a logarithmic age scale at ages $<1 \mathrm{Ga}$, and an age peak $<1 \mathrm{Ga}$ must have a standard deviation of at least $3.5 \%$ of its age value to be fully resolved. For instance, a $100 \mathrm{Ma}$ peak must have a standard deviation of at least 3.5 Ma. An age peak $>1$ Ga must have a standard deviation of at least $35 \mathrm{Ma}$ to be fully resolved. We note that these required widths are somewhat greater than the analytical 
uncertainty of a typical detrital zircon age measurement. However, we suspect that they will be adequate for most detrital zircon age populations, and Figure 2 shows that our implementation completely recovers the age peaks revealed by a popular KDE method (Botev et al., 2010).

Computational constraints are the only limitation on the number of basis functions the method uses. The current default of 100 basis functions distributed on a hybrid log-linear zircon $\mathrm{U}-\mathrm{Pb}$ age axis results in manageable runtimes on a personal computer and resampling experiments suggest that for realistically complex samples (drawn from Pullen et al., 2014), PMEs are not affected by resolution issues. If additional computing power is available, our procedure can easily be altered to use more basis functions and thus resolve narrower and more closely spaced age peaks.

\section{Estimation of BPC uncertainties}

Given the uncertainty inherent in inferring parent population age distributions from detrital zircon samples (Figs. 1, 2), an ideal comparative metric would provide a robust estimate of uncertainty of the metric itself. PMEs contain a large number of potential parent population PDFs, and the variation within PMEs provides a means to estimate BPC uncertainty. In order to estimate BPC uncertainty, we randomly select models from the joint and separate PMEs and compare their likelihood values following Eqn. (6) to calculate a representative set of individual $\Lambda$ values, which we refer to as $\Lambda_{i}$ :

$$
\Lambda_{i}=\ln \mathrm{P}\left(\boldsymbol{d}_{J} \mid \boldsymbol{\theta}_{J, i}\right)-\ln \mathrm{P}\left(\boldsymbol{d}_{1} \mid \boldsymbol{\theta}_{1, i}\right)-\ln \mathrm{P}\left(\boldsymbol{d}_{2} \mid \boldsymbol{\theta}_{2, i}\right)
$$

where $\boldsymbol{\theta}_{J, i}, \boldsymbol{\theta}_{1, i}$, and $\boldsymbol{\theta}_{2, i}$ are models randomly drawn from their respective PMEs. The set of $\Lambda_{i}$ values is then propagated through Eqn. (13) to yield a distribution of BPC values for two samples. The $1 \sigma$ confidence interval of this distribution is the BPC uncertainty $\sigma_{B P C}$. A similar approach to uncertainty estimation in a Bayesian framework is taken by Kruschke (2013).

In order to test the reliability of $\sigma_{B P C}$, we apply it to the synthetic samples used in the previous section (Fig. 9). We calculate $\sigma_{B P C}$ for random subsamples from two well resolved, real detrital zircon age distributions (Pullen et al., 2014; Thomson et al., 2017) at sample sizes of $n=60,117$, and 300 (the same subsamples as shown in Fig. 7). For samples drawn from identical parent populations (Fig. 9a), $\sigma_{B P C}$ proves to be a conservative estimate of uncertainty, with calculated BPC values always lying well within $2 \sigma_{B P C}$ of 
(a)

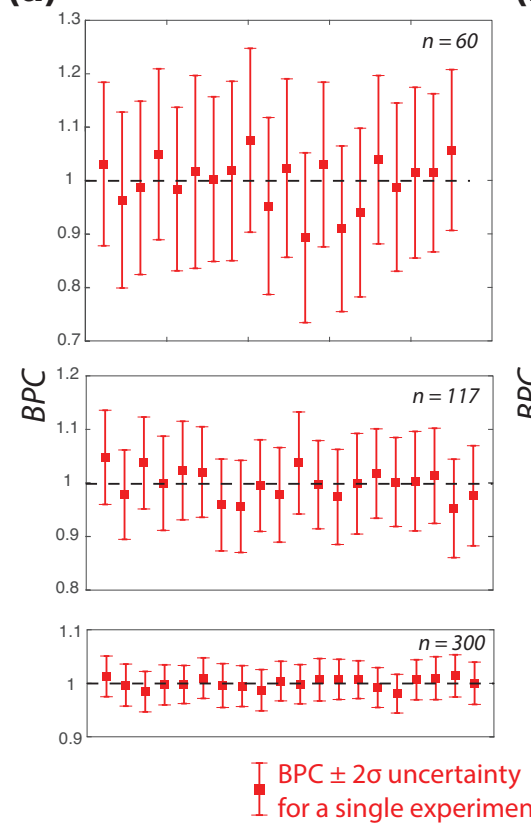

(b)
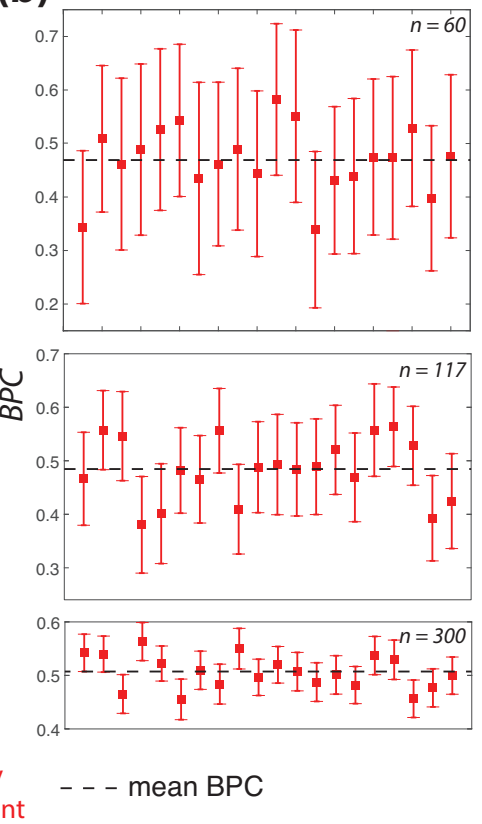

Figure 9: Resampling experiments show that the variation of models within PMEs permits estimation of robust BPC uncertainties. (a) BPC with uncertainties for 20 experiments with random subsamples drawn from identical parent populations (Pullen et al., 2014) and compared against one another. Points show BPC values calculated for two synthetic detrital zircon samples and error bars show uncertainty inferred for that particular pair of samples using the method discussed in Section 5. (b) BPC with estimated uncertainties for 20 experiments where random subsamples were drawn from two different parent populations (Pullen et al., 2014; Thomson et al., 2017). Black dashed line indicates mean $\mathrm{BPC}$ value for each panel, taken as an estimate of the expected BPC value. Uncertainties are calculated from the variation in PMEs (see Section 5). 
the mean value calculated for each sample size. For samples drawn from two different parent populations (Fig. 9b), $\sigma_{B P C}$ is a roughly appropriate estimate of scatter of BPC values about the mean for a given sample size. In this case, for $n=60$, all samples fall within $2 \sigma_{B P C}$ of the mean. For $n$ $=117,85 \%$ of $\mathrm{BPC}$ values fall within $2 \sigma_{B P C}$ of the mean. For $n=300$, $75 \%$ of $\mathrm{BPC}$ values fall within $2 \sigma_{B P C}$ of the mean. Even for those samples further than $2 \sigma_{B P C}$ from the mean, the mean is typically just beyond their $2 \sigma_{B P C}$ error envelope, and $\sigma_{B P C}$ seems to provide a sensible estimate of the scatter in $\mathrm{BPC}$ values for a given sample size. Therefore, we regard $\sigma_{B P C}$ as a useful indicator of BPC uncertainty that reflects the inherent uncertainty in sampling from an unknown parent population.

\section{Implications of PMEs and BPC for analysis of detrital geochronol- ogy data}

We have shown that $\mathrm{BPC}$ is a correspondence metric that varies predictably between 0 and 1, shows minimal or no sample size biasing (Fig. 7), and for which uncertainties can be readily estimated (Fig. 9). These features indicate that $\mathrm{BPC}$ is potentially a more reliable metric of correspondence between detrital zircon populations than other metrics currently available.

We have shown that the functional relationship between BPC and the proportions $\left(f_{1}, f_{2}\right)$ of ages that are shared between two populations can be derived analytically from probability theory (Fig. 8, also see Section S5). This relationship between BPC and the shared proportions of the two populations can be inverted, so that a BPC value calculated for a pair of detrital zircon samples can be used to constrain the shared proportions of their respective parent populations. Because BPC is a function of two independent variables $\left(f_{1}, f_{2}\right.$; see Fig. 8$)$, a BPC value produces nonunique solutions for $f_{1}$ and $f_{2}$. However, if the value of $f_{1}$ or $f_{2}$ can be assumed, then a unique solution for the other is possible. Such an assumption might be able to be made given prior knowledge of the sedimentary system in which the zircons were deposited. For instance, if one sample is collected from a location upstream of another sample, it can be assumed that the entirety of the upstream population is shared with the downstream population, i.e., that it is theoretically possible for any age present in the upstream population to also be present in the downstream population. In such a case, $f_{1}$ can be assumed to equal 1 , permitting the BPC calculated for the two samples to yield a unique solution for $f_{2}$, which constrains the proportion of grains in the downstream popu- 
lation that originated in the catchment of the upstream population. Such a constraint could potentially be used, in combination with data on stream length, catchment area, and erosion rate, and under a set of assumptions, to investigate dilution of zircon age populations in sedimentary systems (see, for example, Niemi, 2013, and Section S5 for further discussion).

We have also shown that a PME is a representative set of the possible parent populations that are likely to have produced a given detrital zircon sample (Section 2). The diversity of PMEs mirrors the diversity of samples of a certain size obtained from a population (Fig. 2). Thus, PMEs may prove useful for the visual assessment of statistical confidence in a detrital zircon sample age distribution. In addition, other types of quantitative analyses besides BPC may be made possible or made more robust through the use of PMEs.

\section{Conclusions}

We develop a metric for comparing two detrital zircon samples-Bayesian Population Correlation (BPC) - that is unbiased with respect to sample size, permits robust estimation of uncertainty, and behaves in a consistent and mathematically predictable manner. Much of the success of this metric depends on the use of a probability model ensemble (PME), rather than a single PDF, to characterize the age distribution of a detrital zircon population. A PME is generated by a Markov Chain Monte Carlo algorithm and is a probabilistically representative set of the potential parent populations consistent with an observed detrital zircon age sample.

Because of the grounding of BPC in probability theory, the shared proportions of two detrital zircon populations can be directly inferred from calculated BPC values. Such inference, along with the ability to estimate robust uncertainties, may permit new quantitative interpretations of detrital zircon age data. In addition, BPC may be applicable beyond detrital zircon data, such as to other types of detrital geochronology or thermochronology data. As shown here, multi-modal datasets with limited sample sizes may not be well-described by existing widely-used statistical methods - in general, these datasets could benefit from BPC analysis.

\section{Acknowledgments}

This work was partially supported by NSF grants EAR-1151247 and EAR-1524304 (NAN). The Turner Fund from the Department of Earth and 
Environmental Sciences at the University of Michigan supported this work through a postdoctoral fellowship to A. S. Wolf and research grant to A. R. Tye. N. Niemi also acknowledges a CIRES Sabbatical Fellowship from the University of Colorado and colleagues from that institution who stimulated the early seeds of this research. We also acknowledge many helpful conversations with Eric Hetland. Scripts for using our procedure can be found at https://github.com/alextye/BPC.

Amidon, W. H., Burbank, D. W., Gehrels, G. E., 2005. U-Pb zircon ages as a sediment mixing tracer in the Nepal Himalaya. Earth and Planetary Science Letters 235 (1), 244-260.

Andersen, T., 2005. Detrital zircons as tracers of sedimentary provenance: Limiting conditions from statistics and numerical simulation. Chemical Geology 216 (3), 249-270.

Botev, Z. I., Grotowski, J. F., Kroese, D. P., et al., 2010. Kernel density estimation via diffusion. The annals of Statistics 38 (5), 2916-2957.

De Boor, C., 1978. A practical guide to splines. Vol. 27. springer-verlag New York.

DeGraaff-Surpless, K., Mahoney, J. B., Wooden, J. L., McWilliams, M. O., 2003. Lithofacies control in detrital zircon provenance studies: Insights from the Cretaceous Methow basin, southern Canadian Cordillera. Geological Society of America Bulletin 115 (8), 899-915.

Dickinson, W. R., 2008. Impact of differential zircon fertility of granitoid basement rocks in North America on age populations of detrital zircons and implications for granite petrogenesis. Earth and Planetary Science Letters 275 (1), 80-92.

Dodson, M., Compston, W., Williams, I., Wilson, J., 1988. A search for ancient detrital zircons in Zimbabwean sediments. Journal of the Geological Society 145 (6), 977-983.

Eilers, P. H., Marx, B. D., 1996. Flexible smoothing with b-splines and penalties. Statistical science, 89-102. 
Fedo, C. M., Sircombe, K. N., Rainbird, R. H., 2003. Detrital zircon analysis of the sedimentary record. Reviews in Mineralogy and Geochemistry 53 (1), 277-303.

Ferguson, T. S., 1962. A representation of the symmetric bivariate cauchy distribution. The Annals of Mathematical Statistics 33 (4), 1256-1266.

Garçon, M., Chauvel, C., 2014. Where is basalt in river sediments, and why does it matter? Earth and Planetary Science Letters 407, 61-69.

Gehrels, G., 2012. Detrital zircon U-Pb geochronology: Current methods and new opportunities. In: Busby, C., Azor, A. (Eds.), Tectonics of Sedimentary Basins: Recent Advances. Blackwell Publishing Ltd., pp. 45-62.

Gehrels, G. E., 2000. Introduction to detrital zircon studies of Paleozoic and Triassic strata in western Nevada and northern California. In: Soreghan, M. J., Gehrels, G. E. (Eds.), Paleozoic and Triassic paleogeography and tectonics of western Nevada and northern California: Geological Society of America Special Paper 347. Geological Society of America, Boulder, Colorado, pp. 1-17.

Gehrels, G. E., Valencia, V. A., Ruiz, J., 2008. Enhanced precision, accuracy, efficiency, and spatial resolution of $\mathrm{U}-\mathrm{Pb}$ ages by laser ablationmulticollector-inductively coupled plasma-mass spectrometry. Geochemistry, Geophysics, Geosystems 9 (3), Q03017.

Gelman, A., Stern, H. S., Carlin, J. B., Dunson, D. B., Vehtari, A., Rubin, D. B., 2013. Bayesian data analysis. Chapman and Hall/CRC.

Geyer, C. J., 1992. Practical Markov chain Monte Carlo. Statistical science, 473-483.

Hanchar, J., Miller, C., 1993. Zircon zonation patterns as revealed by cathodoluminescence and backscattered electron images: implications for interpretation of complex crustal histories. Chemical geology 110 (1-3), $1-13$.

Hurford, A., Fitch, F., Clarke, A., 1984. Resolution of the age structure of the detrital zircon populations of two Lower Cretaceous sandstones from the Weald of England by fission track dating. Geological Magazine 121 (04), 269-277. 
Ibañez-Mejia, M., Pullen, A., Pepper, M., Urbani, F., Ghoshal, G., IbañezMejia, J. C., 2018. Use and abuse of detrital zircon U-Pb geochronology-A case from the Río Orinoco delta, eastern Venezuela. Geology.

Jeffreys, H., 1998. The theory of probability (3rd ed.). Oxford University Press.

Kimbrough, D. L., Grove, M., Gehrels, G. E., Dorsey, R. J., Howard, K. A., Lovera, O., Aslan, A., House, P. K., Pearthree, P. A., 2015. Detrital zircon $\mathrm{U}-\mathrm{Pb}$ provenance of the Colorado River: A 5 my record of incision into cover strata overlying the Colorado Plateau and adjacent regions. Geosphere 11 (6), 1719-1748.

Kruschke, J. K., 2013. Bayesian estimation supersedes the t test. Journal of Experimental Psychology: General 142 (2), 573.

Lange, K. L., Little, R. J., Taylor, J. M., 1989. Robust statistical modeling using the $\mathrm{t}$ distribution. Journal of the American Statistical Association 84 (408), 881-896.

Lawrence, R. L., Cox, R., Mapes, R. W., Coleman, D. S., 2011. Hydrodynamic fractionation of zircon age populations. Geological Society of America Bulletin 123 (1-2), 295-305.

Niemi, N. A., 2013. Detrital zircon age distributions as a discriminator of tectonic versus fluvial transport: An example from the Death Valley, USA, extended terrane. Geosphere 9 (1), 126-137.

Pullen, A., Ibáñez-Mejía, M., Gehrels, G. E., Ibáñez-Mejía, J. C., Pecha, M., 2014. What happens when $n=1000$ ? Creating large-n geochronological datasets with LA-ICP-MS for geologic investigations. Journal of Analytical Atomic Spectrometry 29 (6), 971-980.

Raftery, A. E., 1995. Bayesian model selection in social research. Sociological methodology, 111-163.

Satkoski, A. M., Wilkinson, B. H., Hietpas, J., Samson, S. D., 2013. Likeness among detrital zircon populations-An approach to the comparison of age frequency data in time and space. Geological Society of America Bulletin 125 (11-12), 1783-1799. 
Saylor, J. E., Knowles, J. N., Horton, B. K., Nie, J., Mora, A., 2013. Mixing of source populations recorded in detrital zircon $\mathrm{U}-\mathrm{Pb}$ age spectra of modern river sands. The Journal of Geology 121 (1), 17-33.

Saylor, J. E., Stockli, D. F., Horton, B. K., Nie, J., Mora, A., 2012. Discriminating rapid exhumation from syndepositional volcanism using detrital zircon double dating: Implications for the tectonic history of the Eastern Cordillera, Colombia. Geological Society of America Bulletin 124 (5-6), 762-779.

Saylor, J. E., Sundell, K. E., 2016. Quantifying comparison of large detrital geochronology data sets. Geosphere 12 (1), GES01237-1.

Shanno, D. F., 1970. Conditioning of quasi-newton methods for function minimization. Mathematics of computation 24 (111), 647-656.

Sharman, G. R., Johnstone, S. A., 2017. Sediment unmixing using detrital geochronology. Earth and Planetary Science Letters 477, 183-194.

Sharman, G. R., Sharman, J. P., Sylvester, Z., 2018. detritalpy: A pythonbased toolset for visualizing and analysing detrital geo-thermochronologic data. The Depositional Record 4 (2), 202-215.

Shimazaki, H., Shinomoto, S., 2010. Kernel bandwidth optimization in spike rate estimation. Journal of computational neuroscience 29 (1-2), 171-182.

Silverman, B. W., 1986. Density estimation for statistics and data analysis. Vol. 26. CRC press.

Sircombe, K. N., 2000. Quantitative comparison of large sets of geochronological data using multivariate analysis: A provenance study example from Australia. Geochimica et Cosmochimica Acta 64 (9), 1593-1616.

Spencer, C. J., Kirkland, C. L., 2016. Visualizing the sedimentary response through the orogenic cycle: A multidimensional scaling approach. Lithosphere 8 (1), 29-37.

Sundell, K. E., Saylor, J. E., 2017. Unmixing detrital geochronology age distributions. Geochemistry, Geophysics, Geosystems 18 (8), 2872-2886. 
Thomson, K. D., Stockli, D. F., Clark, J. D., Puigdefàbregas, C., Fildani, A., 2017. Detrital zircon (U-Th)/(He-Pb) double-dating constraints on provenance and foreland basin evolution of the Ainsa Basin, south-central Pyrenees, Spain. Tectonics 36 (7), 1352-1375, 2017TC004504.

Tranel, L. M., Spotila, J. A., Kowalewski, M. J., Waller, C. M., 2011. Spatial variation of erosion in a small, glaciated basin in the Teton Range, Wyoming, based on detrital apatite (U-Th)/He thermochronology. Basin Research 23 (5), 571-590.

Vermeesch, P., 2004. How many grains are needed for a provenance study? Earth and Planetary Science Letters 224 (3), 441-451.

Vermeesch, P., 2012. On the visualisation of detrital age distributions. Chemical Geology 312, 190-194.

Vermeesch, P., 2013. Multi-sample comparison of detrital age distributions. Chemical Geology 341, 140-146. 